\title{
Cytogenetic Partial Response
}

National Cancer Institute

\section{Source}

National Cancer Institute. Cytogenetic Partial Response. NCI Thesaurus. Code C123582.

A partial decrease in abnormal karyotype, based on pre-defined thresholds. 